\title{
Nuclear data needs for decommissioning and design of nuclear facilities
}

\author{
I.C. Sagrado García ${ }^{1, a}$, V. Blideanu ${ }^{1}$, S. Delacroix ${ }^{2}$, J.M. Dumas ${ }^{1}$, D. Ridikas ${ }^{1}$, and A. Van Lauwe ${ }^{1}$ \\ 1 CEA Saclay DSM/DAPNIA, 91191 Gif-sur-Yvette, France \\ 2 Institut Curie-Centre de Proton-Thérapie d'Orsay, France
}

\begin{abstract}
Design and decommissioning of large-scale nuclear facilities is nowadays a high-interest activity for the nuclear community. Nuclear data have an important role in R\&D studies for accelerator-based applications and decommissioning of nuclear facilities including nuclear reactors. Most of these studies use simulations to predict activity levels and dose rates, to design shielding components, etc. The different data libraries and/or reaction models included in the transport codes are essential for these calculations. In this contribution we present several examples of the nuclear data usage for this type of applications. We show the importance of a correct use of nuclear data and reaction models for the accuracy of the results obtained by simulations.
\end{abstract}

\section{Introduction}

Installations able to provide high intensity radioactive beams (SPIRAL2, EURISOL...) are subject of sustained R\&D efforts. There are also an important number of small cyclotrons, most of them dedicated to medical applications, with specific needs in terms of R\&D studies. Decommissioning of nuclear facilities also needs dedicated activity studies. In this work we present two different examples: R\&D studies for acceleratorbased applications and decommissioning of nuclear facilities. Both examples clearly show the role played by nuclear data and their employment in reaction codes for the accuracy of the results obtained by simulations.

\section{R\&D studies for accelerator applications}

Due to progress made in acceleration techniques and targets design, recent years have seen significant growth in the number of small cyclotrons dedicated to light-ion beams in order to produce the radio-isotopes for medical therapy as TAT (Targeted Alpha Therapy) or for PET (Positron Emission Tomography). Accurate estimation of radiation fields at accelerators facilities is an important R\&D related issue. Monte Carlo based simulation tools are very often employed for such studies, mainly due to their capabilities in simulation complex geometries. These codes provide useful, timesaving possibilities to the user as the easy access application related information: particle tracks, prompt dose rates, heat deposition in components, residual inventory, etc. However, one should keep in mind that, behind theses tools, the appropriate description of all nuclear interactions plays a crucial role in the accuracy of the results. In this work we present the study realized for the Orsay Proton-Therapy Center at Curie Institute as an example of R\&D studies for small cyclotrons dedicated to medical applications.

\footnotetext{
a Presenting author,

e-mail: inmaculada.sagradogarcia@cea.fr
}

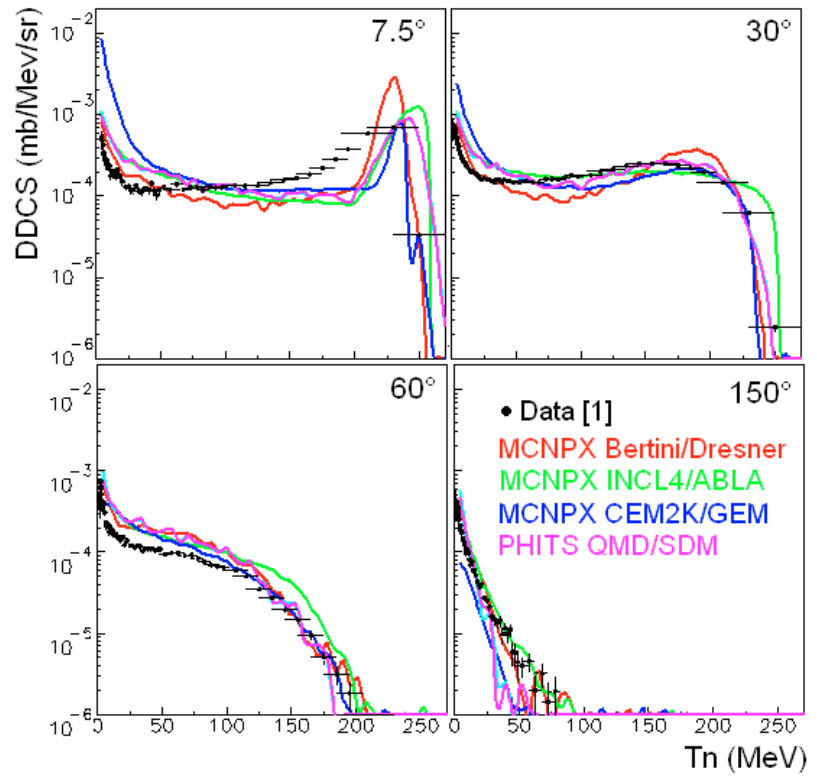

Fig. 1. ${ }^{12} \mathrm{C}(\mathrm{p}, \mathrm{xn})$ double differential cross sections at $256 \mathrm{MeV}$ for 7.5, 30, 60 and 150 degrees. Comparison between calculated values obtained from different reactions models and experimental data from reference [1]. See the text for more details.

\subsection{Orsay proton-therapy centre}

At the Orsay proton-therapy centre at Curie Institute a proton beam is used to treat two types of tumours: brain and eye cancer. The cyclotron delivers a $200 \mathrm{MeV}$ proton beam, which is well adapted for the brain cancer treatment. However for the eye cancer treatment a $73 \mathrm{MeV}$ proton beam is needed. To slow down the protons from 200 to $73 \mathrm{MeV}$ a graphite degrader is used. Therefore, an important number of neutrons are emitted due to ${ }^{12} \mathrm{C}(\mathrm{p}, \mathrm{xn})$ reactions at $200 \mathrm{MeV}$. To restrict the dose rate in the places close to the degrader, the Curie Institute wishes to built a local shielding in the degrader room. The aim of this work is to determine the most appropriate shielding for this particular case in terms of materials and wall thicknesses. 


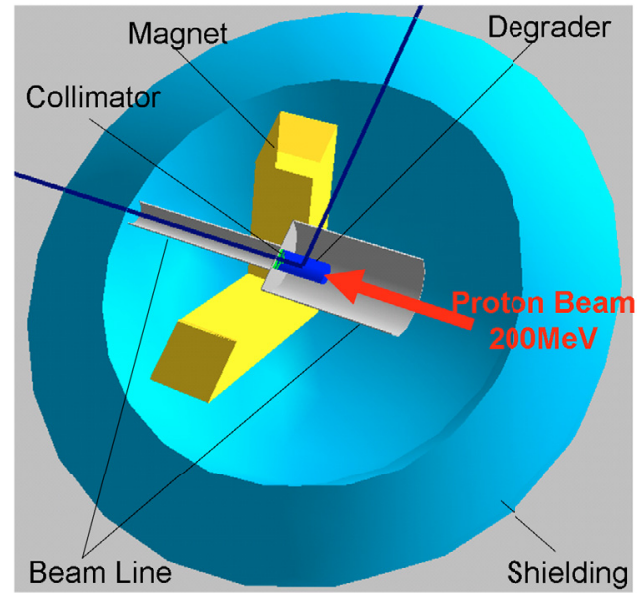

Fig. 2. Geometry of degrader assembly used in simulations.

In this work we are interested in the ${ }^{12} \mathrm{C}(\mathrm{p}, \mathrm{xn})$ reactions at $200 \mathrm{MeV}$. The most appropriate experimental data available are the ${ }^{12} \mathrm{C}(\mathrm{p}, \mathrm{xn})$ double differential cross sections at $256 \mathrm{MeV}$ measured for several angles by Meier [1]. In order to select the reaction models to be used for the simulations we have calculated the ${ }^{12} \mathrm{C}(\mathrm{p}, \mathrm{xn})$ double differential cross sections at $256 \mathrm{MeV}$ coupling different evaporation (Dresner, ABLA, GEM, SDM [2-5]) and intranuclear cascade (Bertini, INCL4, CEM2K, QMD [6-9]) models included in the transport codes MCNPX [10] and PHITS [11]. Figure 1 shows the comparison between calculations and experimental data. In ideal situation, one desires that the selected model reproduces the measurements as closely as possible. On other hand, keeping in mind that the objective is the shielding definition, the model must not under-estimate the data, in particular for elastic and evaporation processes, responsible for the major contribution at forward and backward angles respectively. According to these criteria the SDM evaporation model coupled with QMD cascade model will be employed for the following simulations. Both models are included in the PHITS transport code.

After the model selection the geometry of the degrader assembly has been modelled using the MORITZ package [12] (fig. 2). This geometry, together with SDM and QMD models, has been employed to perform the PHITS transport calculations.

To determine the principal characteristics of the future shielding (material and thickness) a spherical geometry is used (fig. 2). This simplified geometry allows determining the shielding efficiency for emitted neutrons in all directions. Shielding efficiency is directly related to the emitted neutron energy. In ${ }^{12} \mathrm{C}(\mathrm{p}, \mathrm{xn})$ reactions low energy neutrons are principally produced in evaporation process. This process is characterized by an isotropic emission, while energetic neutrons produced in direct processes are mainly emitted at forward angles (fig. 1).

In order to get reference values for neutron dose rate, the first simulation has been realized without shielding. The next step consists of performing subsequent simulations using different shielding combinations. Due to limited space of this manuscript, only two of them are presented in this contribution: one is using $1 \mathrm{~m}$ of concrete and the other a combination

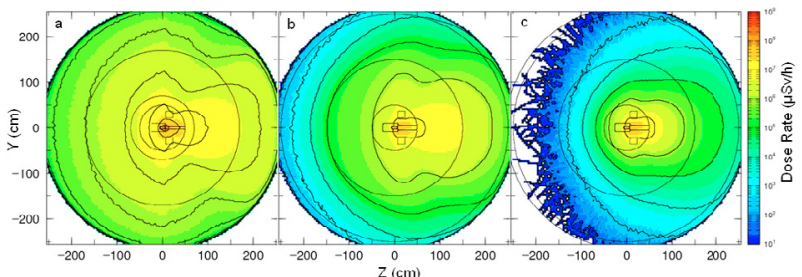

Fig. 3. Dose rate distributions around the degrader assembly for different shielding combinations: without shielding (a), $1 \mathrm{~m}$ of concrete (b), $1 \mathrm{~m}$ of concrete $+1 \mathrm{~m}$ of heavy concrete (c).
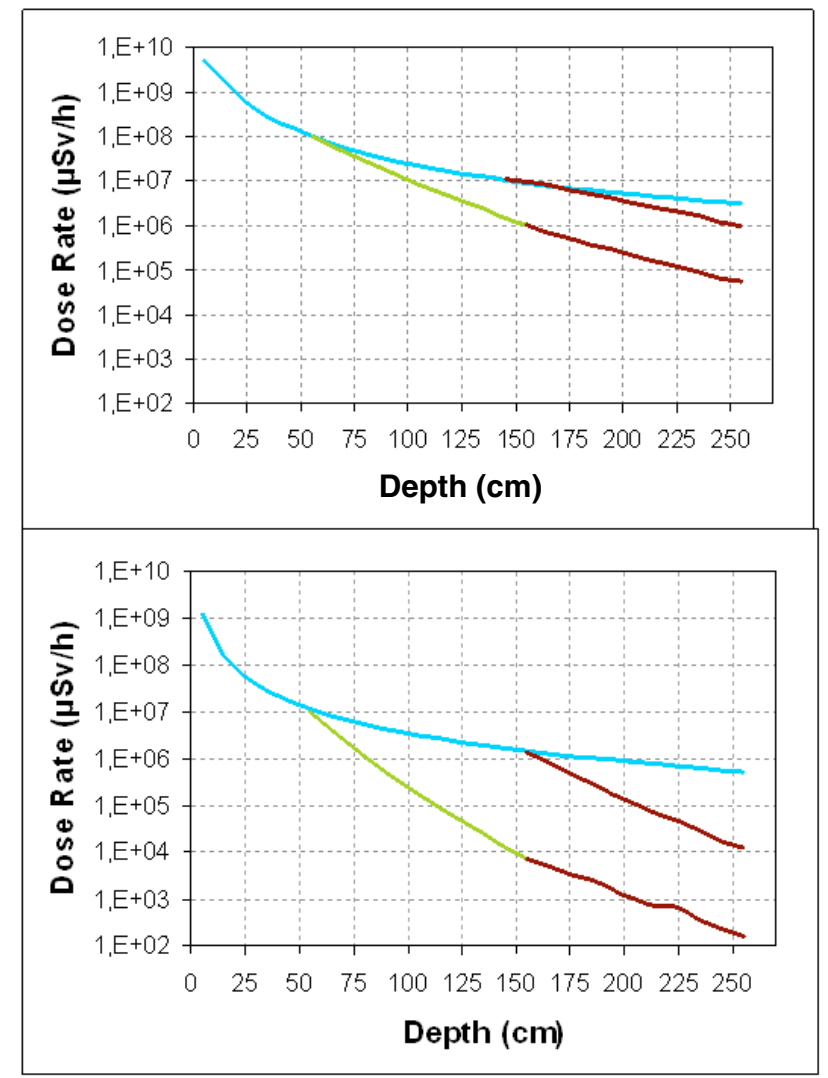

Fig. 4. Shielding efficiency in depth for different traversed materials, air (blue), concrete (brown) and heavy concrete (green).

of $1 \mathrm{~m}$ of concrete and $1 \mathrm{~m}$ of heavy concrete. Obtained dose rate distributions for these three simulations are presented in figure 3.

Shielding efficiency in shielding depth has also been calculated for different directions. Obtained results at 0 and 90 degrees are presented in figure 4, upper and lower panel respectively. As expected, the dose rate is more important in the beam direction due to contribution of the most energetic neutrons. At $250 \mathrm{~cm}$ from the degrader, $1 \mathrm{~m}$ of concrete slightly reduces the dose rate at 0 degrees. On the other hand, $1 \mathrm{~m}$ of concrete used together with $1 \mathrm{~m}$ of heavy concrete reduces the dose rate by two and three orders of magnitude at 0 and 90 degrees respectively.

During brain cancer treatments, the proton beam delivered by the cyclotron arrives directly on the brass collimator. The interaction of $200 \mathrm{MeV}$ protons with the different elements of 


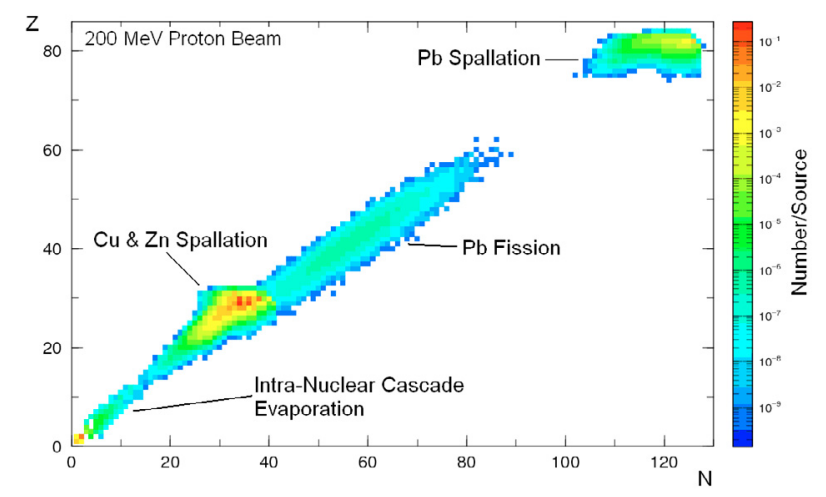

Fig. 5. Produced isotopes from $200 \mathrm{MeV}$ proton induced reactions in collimator during the brain cancer treatments.

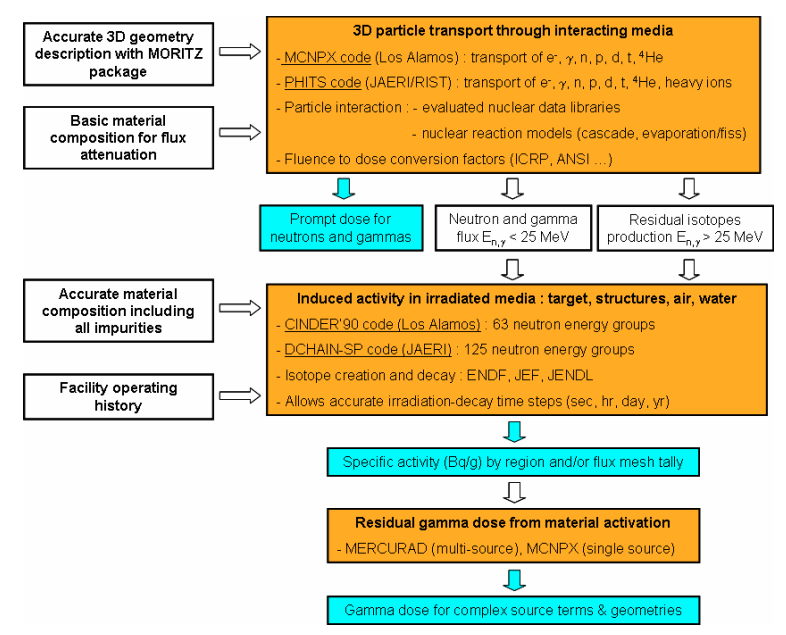

Fig. 6. Methodology used for activation studies.
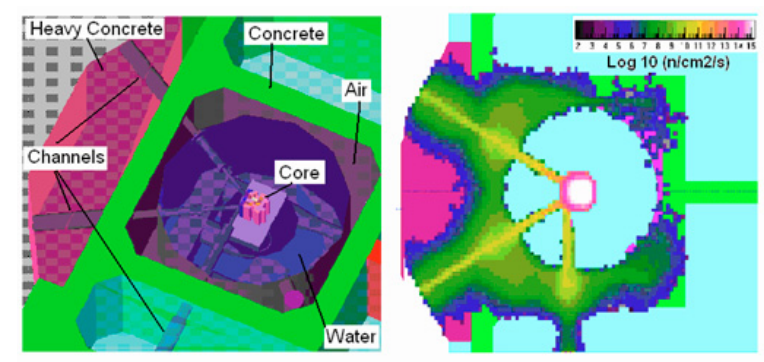

Fig. 7. SILOE reactor: geometry used in simulations (left) and calculated 3D neutron distribution (right).

the collimator, mainly copper, zinc and lead, produces a large variety of isotopes. Figure 5 shows the results obtained from simulations. We observe the isotopes produced by spallation and fission reactions on lead, spallation reactions on zinc and copper and the light isotopes created in intra-nuclear cascade and evaporation processes.

\section{Decommissioning of nuclear facilities}

Decommissioning of large nuclear facilities is not only a technical but also an economical challenge due to the hundreds of tones of structural and shielding material to deal with.

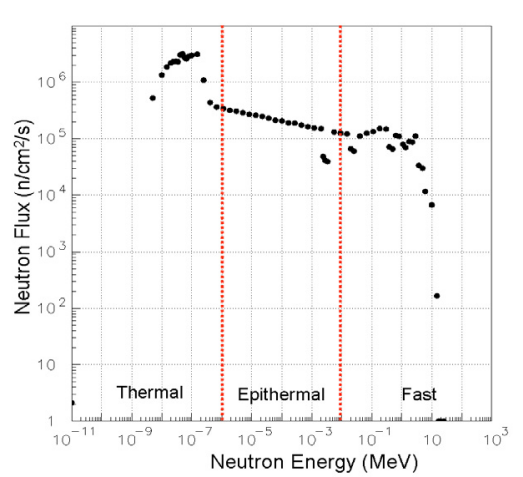

Fig. 8. Calculated 63-group neutron flux used in CINDER'90.

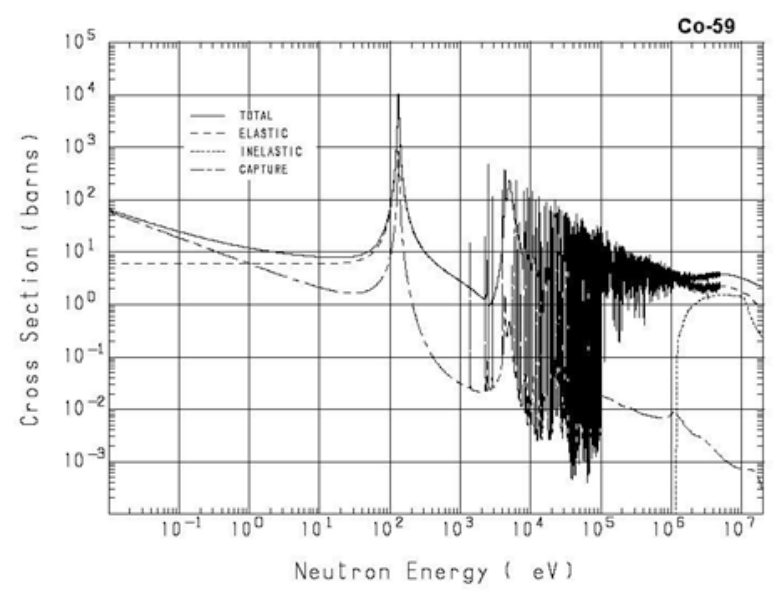

Fig. 9. Cross sections for neutron induced reactions on ${ }^{59} \mathrm{Co}$.

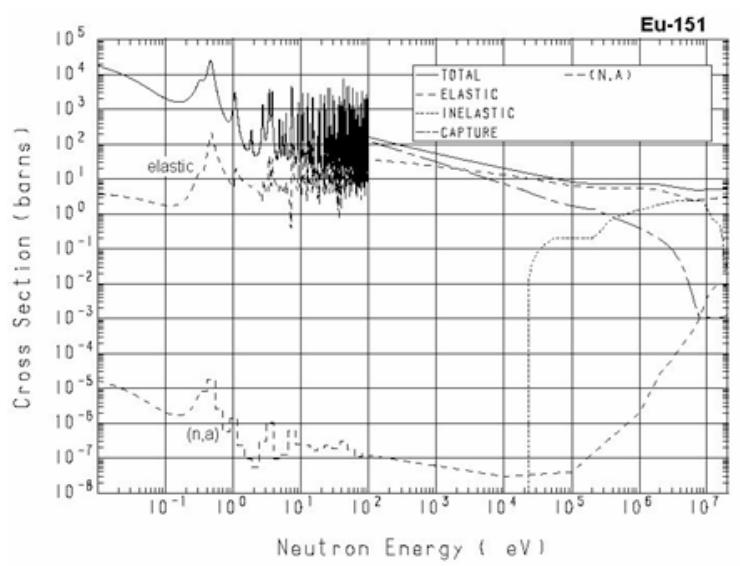

Fig. 10. Cross sections for neutron induced reactions on ${ }^{151} \mathrm{Eu}$.

Optimization of mass flow to different waste repositories is a critical issue since the storage cost is depending on the waste activation level. In order to provide a complete description of the activity induced by neutrons during the facility operation we developed a method based on Monte Carlo calculations witch uses MCNPX [10] or PHITS [11] codes for particle transport and CINDER'90 [13] or DCHAIN [14] codes for activation calculations (see fig. 6). The method has been successfully used for several French reactors from $100 \mathrm{~kW}$ to 


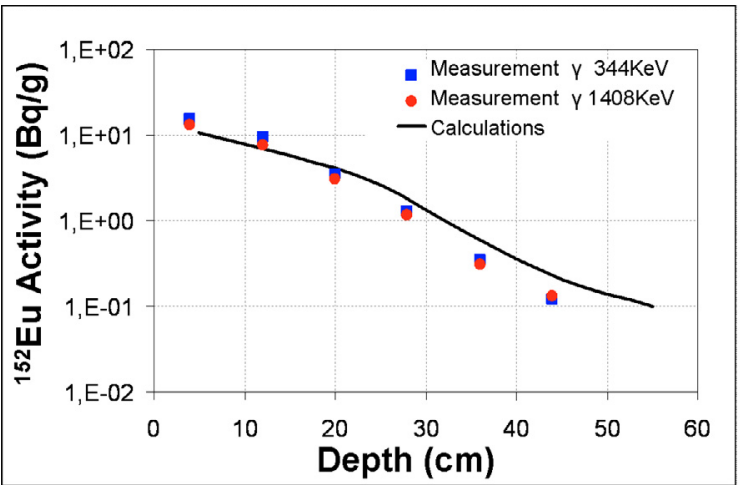

Fig. 11. Activity of ${ }^{152}$ Eu: comparison between calculated and experimental values for concrete activation in a selected zone of the SILOE reactor.

$40 \mathrm{MW}$ thermal power and other studies being currently under way. In this section we present the study for the SILOE reactor as an example showing the complete procedure of activation studies for nuclear facilities.

\subsection{Activation studies: the SILOE reactor}

The research pool-type reactor SILOE $\left(40 \mathrm{MW}_{\text {th }}\right)$ is located in Grenoble (France). After 35 years of operation it was shutdown in 1997. The aim of this study is to determine the neutron induced activation in the different components of the installation: structural materials, shielding, etc. After modelling the accurate $3 \mathrm{D}$ geometry of the installation using the MORITZ package [12] (left part of fig. 7), the MCNPX code has been employed for criticality and neutron transport calculations.

In the SILOE reactor, fission neutrons are efficiently moderated, so most of them become thermal. Therefore the neutron flux distribution (right part of fig. 7) has been calculated using the available cross sections $(\mathrm{En}<20 \mathrm{MeV})$ from the nuclear data libraries ENDF [15] and JEFF [16] included in MCNPX.

The deterministic material evolution code CINDER'90 [13] has been used further to obtain the activity in the selected regions. CINDER'90 needs as input the accurate description of the material composition (including the impurities) the calculated neutron flux using 63 energy groups (see for example fig. 8) and an appropriate set of activation cross sections $($ En $<25 \mathrm{MeV})$. Figures 9 and 10 show the cross sections for ${ }^{59} \mathrm{Co}$ and ${ }^{151} \mathrm{Eu}$, two of the most important contributors in steel and concrete activations respectively.

The final result is a complete distribution of the specific activity for all materials in the selected regions. These results will be used to separate between different waste categories depending on their activation level.

The last and very important step of the procedure consists of the result validation. Obviously the direct comparison between experimental measurements and calculations is not always possible. However, for the case presented in this work, several samples have been taken to measure the activity level. Figure 11 shows the comparison between calculations and measurements for specific activity from ${ }^{152} \mathrm{Eu}$, one of the most important contributors in concrete activation. Keeping in mind that experimental data are given with an uncertainty of about $15 \%$, the agreement found between calculated and experimental values is satisfactory.

\section{Conclusion}

Nuclear data and appropriate description of different nuclear processes are of a great importance in the accuracy of the results obtained by simulations for the design of acceleratorbased applications as well as for decommissioning of nuclear facilities including nuclear reactors.

Therefore the prediction power of reaction models must be systematically tested against experimental data for nuclear reactions of importance in the given application. The accuracy of the results is directly related to the neutron transport across traversed materials, witch is governed by neutron interactions with hundreds of target nuclei. The use of appropriate and validated cross sections for neutron interactions over a wide range of elements is therefore crucial.

\section{References}

1. M.M. Meier et al., Nuc. Sci. Eng. 110 (1992).

2. L. Dresner, Oak Ridge report ORNL-TM-196 (1962).

3. A.R. Junghans et al., Nucl. Phys. A 629 (1998).

4. S. Furihata, Nucl. Instrum. Meth. Phys. Res. B, 171 (2000).

5. O. Iwaqmoto et al., J. Nucl. Sci. Technol., Suppl. 2 (2002).

6. H.W. Bertini, Phys. Rev. 131 (1963).

7. A. Boudard et al., Phys. Rev. C 66 (2002).

8. S.G. Mashnik, A.J. Sierk, Los Alamons National Laboratory report LA-UR-01-5390, and International Conference on Nuclear Data for Science and Technology, Tsukuba, Japan, 2001.

9. K. Niita et al., Phys. Rev. C 52 (1995).

10. D.B. Pelowitz et al., MCNPX User's Manual Version 2.5.0 (2005).

11. H. Iwase et al., Nucl. Instrum. Meth. Phys. Res. B 183, 374 (2001).

12. K.A. Van Riper, Moritz, An Interactive Geometry Editor/Viewer for $M C N P \mathcal{E} M C N P X$, www.whiterockscience.com/moritz.html.

13. W.B. Wilson, T.R. England, K.A. Van Riper, Status of CINDER '90 Codes and Data, Los Alamos National Laboratory, Preprint LA-UR-99-361 (1999).

14. H. Takada, K. Kosako, DCHAIN-SP code, JAERI-Data/Code 99-008.

15. ENDF: Evaluated Nuclear Reaction Data File (2005), www.nndc.bnl.gov/endf.

16. JEFF: Joint Evaluated Fusion and Fission File (2005), www.nea.fr/html/dbdata/projects/nds_jef.htm. 